\title{
Towards a Fuzzy Description Logic for the Semantic Web (Preliminary Report)
}

\author{
U. Straccia \\ ISTI-CNR, Via G. Moruzzi 1, I-56124 Pisa, Italy \\ straccia@isti.cnr.it
}

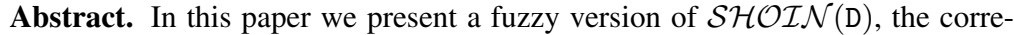
sponding Description Logic of the ontology description language OWL DL. We show that the representation and reasoning capabilities of fuzzy $\mathcal{S H O I N}$ (D)

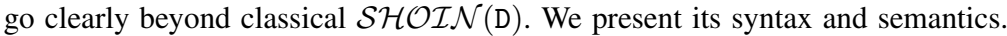
Interesting features are that concrete domains are fuzzy and entailment and subsumption relationships may hold to some degree in the unit interval $[0,1]$.
\end{abstract}

\section{Introduction}

In the last decade a substantial amount of work has been carried out in the context of Description Logics (DLs) [2]. DLs are a logical reconstruction of the so-called framebased knowledge representation languages, with the aim of providing a simple wellestablished Tarski-style declarative semantics to capture the meaning of the most popular features of structured representation of knowledge.

Nowadays, DLs have gained even more popularity due to their application in the context of the Semantic Web $[4,15]$. Semantic Web has recently attracted much attention both from academia and industry, and is widely regarded as the next step in the evolution of the World Wide Web. It aims at enhancing content on the World Wide Web with meta-data, enabling agents (machines or human users) to process, share and interpret Web content.

Ontologies [10] play a key role in the Semantic Web and major effort has been put by the Semantic Web community into this issue. Informally, an ontology consists of a hierarchical description of important concepts in a particular domain, along with the description of the properties (of the instances) of each concept. DLs play a particular role in this context as they are essentially the theoretical counterpart of the Web Ontology Language $O W L D L$, the state of the art language to specify ontologies. Web content is then annotated by relying on the concepts defined in a specific domain ontology.

However, OWL DL becomes less suitable in all those domains in which the concepts to be represent have not a precise definition. If we take into account that we have to deal with Web content, then it is easily verified that this scenario is, unfortunately, likely the rule rather than an exception. For instance, just consider the case we would like to build an ontology about flowers. Then we may encounter the problem of representing 
concepts like 1 "Candia is a creamy white rose with dark pink edges to the petals", "Jacaranda is a hot pink rose", "Calla is a very large, long white flower on thick stalks". As it becomes apparent such concepts hardly can be encoded into OWL DL, as they involve so-called fuzzy or vague concepts, like "creamy", "dark", "hot", "large" and "thick", for which a clear and precise definition is not possible. ${ }^{2}$

The problem to deal with imprecise concepts has been addressed several decades ago by Zadeh [31], which gave bird in the meanwhile to the so-called fuzzy set and fuzzy logic theory and a huge number of real life applications exists. Unfortunately, despite the popularity of fuzzy set theory, relative little work has been carried out in extending DLs towards the representation of imprecise concepts, notwithstanding DLs can be considered as a quite natural candidate for such an extension $[5,6,13,23,25,26$, 27, 29, 30] (see also [9], Chapter 6).

In this paper we consider a fuzzy extension of $\mathcal{S H O I N}(\mathrm{D})$, the corresponding DL of the ontology description language OWL DL, and present its syntax and semantics. The main feature of fuzzy $\mathcal{S H O I N}(\mathrm{D})$ is that it allows us to represent and reason about vague concepts. None of the approaches to fuzzy DLs deal with the expressive power of the fuzzy extension of $\mathcal{S H O I N}(\mathrm{D})$ we present here. Our purpose is also to integrate most of these contributions within an unique setting and, thus, hope to define

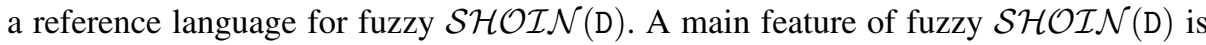
that the subsumption relation between classes and the entailment relation is no more a crisp yes/no problem, but it becomes now fuzzy, i.e. is established to some degree.

We will proceed as follows. In the following section we recall the description logic $\mathcal{S H O I N}(\mathrm{D})$. In Section 3 we extend $\mathcal{S H O \mathcal { N }}$ (D) to the fuzzy case and discuss some properties of it. Section 4 concludes and presents some topics for further research.

\section{Preliminaries}

The ontology language OWL DL is a syntactic variant of $\mathcal{S H O I N}$ (D) [15]. Although several XML and RDF syntaxes for OWL-DL exist, in this paper we use the traditional description logic notation. For explicating the relationship between OWL DL and DLs syntax, see e.g. $[15,16]$. The purpose of this section is to make the paper selfcontained. More importantly it helps in understanding the differences between classical $\mathcal{S H O I N}(\mathrm{D})$ and fuzzy $\mathcal{S H O I N}(\mathrm{D})$. The reader confident with the $\mathcal{S H O I N}$ (D) terminology may skip directly to Section 3.

Syntax. $\mathcal{S H O I N}(\mathrm{D})$ allows to reason with concrete data types, such as strings and integers using so-called concrete domains [1, 18, 20,21]. A concrete domain $\mathrm{D}$ is a pair $\left\langle\Delta_{\mathrm{D}}, \Phi_{\mathrm{D}}\right\rangle$, where $\Delta_{\mathrm{D}}$ is an interpretation domain and $\Phi_{\mathrm{D}}$ is the set of concrete domain predicates $d$ with a predefined arity $n$ and an interpretation $d^{\mathrm{D}} \subseteq \Delta_{\mathrm{D}}^{n}$. For instance, over the integers $\geq_{20}$ may be an unary predicate denoting the set of integers greater or

\footnotetext{
${ }^{1}$ Taken from a text book on flowers.

${ }^{2}$ Another issue relates to the representation of terms like "very", which are called fuzzy concepts modifiers, as we will see later on.
} 
equal to 20. For instance, Person $\sqcap \exists$ age. $\geq_{20}$ denotes a person whose age is greater or equal to 20 . So, let $\mathrm{C}, \mathrm{R}_{a}, \mathrm{R}_{c}, \mathrm{I}_{a}$ and $\mathrm{I}_{c}$ be non-empty finite and pair-wise disjoint sets of concepts names, abstract roles names, concrete roles names, abstract individual names and concrete individual names. An abstract role is an abstract role name or the inverse $S^{-}$of an abstract role name $S$ (concrete role names do not have inverses). An RBox $\mathcal{R}$ consists of a finite set of transitivity axioms $\operatorname{trans}(R)$, and role inclusion axioms of the form $R \sqsubseteq S$ and $T \sqsubseteq U$, where $R$ and $S$ are abstract roles, and $T$ and $U$ are concrete roles. The reflexive-transitive closure of the role inclusion relationship is denoted with $\sqsubseteq^{*}$. A role not having transitive sub-roles is called simple role. The set of $\mathcal{S H O I N}$ (D) concepts is defined by the following syntactic rules, where $A$ is an atomic concept, $R$ is an abstract role, $S$ is an abstract simple role, $T_{i}$ are concrete roles, $d$ is a concrete domain predicate, $a_{i}$ and $c_{i}$ are abstract and concrete individuals, respectively, and $n \in \mathbb{N}$ :

$$
\begin{aligned}
C \longrightarrow & \top|\perp| A\left|C_{1} \sqcap C_{2}\right| C_{1} \sqcup C_{2}|\neg C| \forall R . C|\exists R . C| \geq n S|\leq n S| \\
& \left\{a_{1}, \ldots, a_{n}\right\}|\geq n T| \leq n T\left|\forall T_{1}, \ldots, T_{n} . D\right| \exists T_{1}, \ldots, T_{n} . D \\
D \longrightarrow & d \mid\left\{c_{1}, \ldots, c_{n}\right\}
\end{aligned}
$$

For instance, we may write the concept Flower $\sqcap$ ( $\exists$ hasPetalWidth. $\left(\geq_{20 \mathrm{~mm}} \sqcap \leq_{40 \mathrm{~mm}}\right.$ )) $\sqcap$ hasColour.Red) to informally denote the set of flowers having petal's dimension within $20 \mathrm{~mm}$ and $40 \mathrm{~mm}$, whose colour is red. Here $\geq_{20 \mathrm{~mm}}$ (and $\leq_{40 \mathrm{~mm}}$ ) is a concrete domain predicate. We use $(=1 S)$ as an abbreviation for $(\geq 1 S) \sqcap(\leq 1 S)$. A TBox $\mathcal{T}$ consists of a finite set of concept inclusion axioms $C \sqsubseteq D$, where $C$ and $D$ are concepts. For ease, we use $C=D \in \mathcal{T}$ in place of $C \sqsubseteq D, D \sqsubseteq C \in \mathcal{T}$. An ABox $\mathcal{A}$ consists of a finite set of concept and role assertion axioms and individual (in)equality axioms $a: C,(a, b): R,(a, c): T, a \approx b$ and $a \not z b$, respectively. A SHOIN $(\mathrm{D})$ knowledge base $\mathcal{K}=\langle\mathcal{T}, \mathcal{R}, \mathcal{A}\rangle$ consists of a TBox $\mathcal{T}$, a $\operatorname{RBox} \mathcal{R}$, and an ABox $\mathcal{A}$.

Semantics. An interpretation $\mathcal{I}$ with respect to a concrete domain $\mathrm{D}$ is a pair $\mathcal{I}=$ $\left(\Delta^{\mathcal{I}},{ }^{\mathcal{I}}\right)$ consisting of a non empty set $\Delta^{\mathcal{I}}$ (called the domain), disjoint from $\Delta_{\mathrm{D}}$, and of an interpretation function. ${ }^{\mathcal{I}}$ that assigns to each $C \in \mathrm{C}$ a subset of $\Delta^{\mathcal{I}}$, to each $R \in \mathrm{R}_{a}$ a subset of $\Delta^{\mathcal{I}} \times \Delta^{\mathcal{I}}$, to each $a \in \mathrm{I}_{a}$ an element in $\Delta^{\mathcal{I}}$, to each $c \in \mathrm{I}_{c}$ an element in $\Delta_{\mathrm{D}}$, to each $T \in \mathrm{R}_{c}$ a subset of $\Delta^{\mathcal{I}} \times \Delta_{\mathrm{D}}$ and to each $n$-ary concrete predicate $d$ the interpretation $d^{\mathrm{D}} \subseteq \Delta_{\mathrm{D}}^{n}$. The mapping $\cdot{ }^{\mathcal{I}}$ is extended to concepts and roles as usual: $\top^{\mathcal{I}}=\Delta^{\mathcal{I}}, \perp^{\mathcal{I}}=\emptyset$,

$$
\begin{aligned}
\left(C_{1} \sqcap C_{2}\right)^{\mathcal{I}} & =C_{1}^{\mathcal{I}} \cap C_{2}{ }^{\mathcal{I}} \\
\left(C_{1} \sqcup C_{2}\right)^{\mathcal{I}} & =C_{1}{ }^{\mathcal{I}} \cup C_{2}{ }^{\mathcal{I}} \\
(\neg C)^{\mathcal{I}} & =\Delta^{\mathcal{I}} \backslash C^{\mathcal{I}} \\
\left(S^{-}\right)^{\mathcal{I}} & =\left\{\langle y, x\rangle:\langle x, y\rangle \in S^{\mathcal{I}}\right\} \\
(\forall R . C)^{\mathcal{I}} & =\left\{x \in \Delta^{\mathcal{I}}: R^{\mathcal{I}}(x) \subseteq C^{\mathcal{I}}\right\} \\
(\exists R . C)^{\mathcal{I}} & =\left\{x \in \Delta^{\mathcal{I}}: R^{\mathcal{I}}(x) \cap C^{\mathcal{I}} \neq \emptyset\right\} \\
(\geq n S)^{\mathcal{I}} & =\left\{x \in \Delta^{\mathcal{I}}:\left|S^{\mathcal{I}}(x)\right| \geq n\right\} \\
(\leq n S)^{\mathcal{I}} & =\left\{x \in \Delta^{\mathcal{I}}:\left|S^{\mathcal{I}}(x)\right| \leq n\right\} \\
\left\{a_{1}, \ldots, a_{n}\right\}^{\mathcal{I}} & =\left\{a_{1}{ }^{\mathcal{I}}, \ldots, a_{n}{ }^{\mathcal{I}}\right\}
\end{aligned}
$$


and similarly for the other constructs, where $R^{\mathcal{I}}(x)=\left\{y:\langle x, y\rangle \in R^{\mathcal{I}}\right\}$ and $|X| \mathrm{de}$ notes the cardinality of the set $X$. In particular,

$$
\left(\exists T_{1}, \ldots, T_{n} . d\right)^{\mathcal{I}}=\left\{x \in \Delta^{\mathcal{I}}:\left[T_{1}^{\mathcal{I}}(x) \times \ldots \times T_{n}^{\mathcal{I}}(x)\right] \cap d^{\mathrm{D}} \neq \emptyset\right\} .
$$

The satisfiability of an axiom $E$ in an interpretation $\mathcal{I}=\left(\Delta^{\mathcal{I}},{ }^{\mathcal{I}}\right)$, denoted $I \models E$, is defined as follows: $I=C \sqsubseteq D$ iff $C^{\mathcal{I}} \subseteq D^{\mathcal{I}}, I \models R \sqsubseteq S$ iff $R^{\mathcal{I}} \subseteq S^{\mathcal{I}}, I=T \sqsubseteq U$ iff $T^{\mathcal{I}} \subseteq U^{\mathcal{I}}, I \models \operatorname{trans}(R)$ iff $R^{\mathcal{I}}$ is transitive, $I \models a: C$ iff $a^{\mathcal{I}} \in C^{\mathcal{I}}, I \models(a, b): R$ iff $\left\langle a^{\mathcal{I}}, b^{\mathcal{I}}\right\rangle \in R^{\mathcal{I}}, I \models(a, c): T$ iff $\left\langle a^{\mathcal{I}}, c^{\mathcal{I}}\right\rangle \in T^{\mathcal{I}}, I \models a \approx b$ iff $a^{\mathcal{I}}=b^{\mathcal{I}}, I \models a \not z b$ iff $a^{\mathcal{I}} \neq b^{\mathcal{I}}$. An abstract simple role $S$ is called functional if the interpretation of role $S$ is always functional. A functional role $S$ can always be obtained from an abstract role by means of the axiom $T \sqsubseteq(\leq 1 S)$. Therefore, whenever we say that a role is functional, we assume that $T \sqsubseteq(\leq 1 S)$ is in the ABox. For a set of axioms $\mathcal{E}$, we say that $I$ satisfies $\mathcal{E}$ iff $I$ satisfies each element in $\mathcal{E}$. If $I \models E$ (resp. $I \models \mathcal{E}$ ) we say that $I$ is a model of $E$ (resp. $\mathcal{E}$ ). I satisfies (is a model of) a knowledge base $\mathcal{K}=\langle\mathcal{T}, \mathcal{R}, \mathcal{A}\rangle$, denoted $I \models \mathcal{K}$, iff $I$ is a model of each component $\mathcal{T}, \mathcal{R}$ and $\mathcal{A}$, respectively. An axiom $E$ is a logical consequence of a knowledge base $\mathcal{K}$, denoted $\mathcal{K} \models E$ iff every model of $\mathcal{K}$ satisfies $E$. According to [16], the entailment and subsumption problem can be reduced to knowledge base satisfiability problem, for which decision procedures and reasoning tools exists (e.g. RACER [11] and FACT [14]).

Example 1. Let us consider the following excerpt of a simple ontology (TBox $\mathcal{T}$ ) about cars, with empty $\operatorname{RBox}(\mathcal{R}=\emptyset)$ :

$$
\begin{array}{cc}
\text { Car } \sqsubseteq(=1 \text { maker }) \sqcap(=1 \text { passanger }) \sqcap(=1 \text { speed }) \\
(=1 \text { maker }) \sqsubseteq \text { Car } & \top \sqsubseteq \forall \text { maker.Maker } \\
(=1 \text { passanger }) \sqsubseteq \text { Car } & \top \sqsubseteq \forall \text { passanger. } \mathbb{N} \\
(=1 \text { speed }) \sqsubseteq \text { Car } & \top \sqsubseteq \forall \text { speed.Km } / \mathrm{h} \\
\text { Roadster } \sqsubseteq \text { Cabriolet } \sqcap \exists \text { passenger. }\{2\} \\
\text { Cabriolet } \sqsubseteq \text { Car } \sqcap \exists \text { topType.SoftTop } \\
\text { SportsCar }=\text { Car } \sqcap \exists \text { speed. } \geq 245 \mathrm{~km} / \mathrm{h}
\end{array}
$$

In $\mathcal{T}$, the value for speed ranges over the concrete domain of kilometers per hour, $\mathrm{Km} / \mathrm{h}$, while the value for passengers ranges over the concrete domain of natural numbers, $\mathbb{N}$. The concrete predicate $\geq_{245 \mathrm{~km} / \mathrm{h}}$ is true if the value is greater or equal than to $245 \mathrm{~km} / \mathrm{h}$. The ABox $\mathcal{A}$ contains the following assertions:

$$
\begin{aligned}
& \text { mgb:Roadster } \sqcap(\exists \text { maker. }\{\text { mg }\}) \sqcap(\exists \text { speed. }\{170 \mathrm{~km} / \mathrm{h}\}) \\
& \text { enzo:Car } \sqcap(\exists \text { maker. }\{\text { ferrari }\}) \sqcap\left(\exists \text { speed. }>_{350 \mathrm{~km} / \mathrm{h}}\right) \\
& \text { tt:Car } \sqcap(\exists \text { maker. }\{\text { audi }\}) \sqcap(\exists \text { speed. }\{243 \mathrm{~km} / \mathrm{h}\})
\end{aligned}
$$

Consider the knowledge base $\mathcal{K}=\langle\mathcal{T}, \mathcal{R}, \mathcal{A}\rangle$. It is then easily verified that, e.g.

$$
\begin{array}{ll}
\mathcal{K} \models \text { Roadster } \sqsubseteq \text { Car } & \mathcal{K} \models \text { mg:Maker } \\
\mathcal{K} \models \text { enzo:SportsCar } & \mathcal{K} \models \text { tt: } \neg \text { SportsCar } .
\end{array}
$$


The above example illustrates an evident difficulty in defining the class of sport cars. Indeed, it is highly questionable why a car whose speed is $243 \mathrm{~km} / \mathrm{h}$ is not a sport car any more. The point is that essentially, the higher the speed the more likely a car is a sports car, which makes the concept of sports car rather a fuzzy concept, i.e. vague concept, rather than a crisp one. In the next section we will see how to represent such concepts more appropriately.

\section{Fuzzy OWL DL}

Fuzzy sets have been introduced by Zadeh [31] as a way to deal with vague concepts like low pressure, high speed and the like. Formally, a fuzzy set $A$ with respect to a universe $X$ is characterized by a membership function $\mu_{A}: X \rightarrow[0,1]$, assigning an $A$-membership degree, $\mu_{A}(x)$, to each element $x$ in $X . \mu_{A}(x)$ gives us an estimation of the belonging of $x$ to $A$. Typically, if $\mu_{A}(x)=1$ then $x$ definitely belongs to $A$, while $\mu_{A}(x)=0.8$ means that $x$ is "close" to be an element of $A$.

When we switch to fuzzy logics, the notion of degree of membership $\mu_{A}(x)$ of an element $x \in X$ w.r.t. the fuzzy set $A$ over $X$ is regarded as the degree of truth in $[0,1]$ of the statement " $x$ is $A$ ". Accordingly, in our fuzzy DL, $(i)$ a concept $C$, rather than being interpreted as a classical set, will be interpreted as a fuzzy set and, thus, concepts become imprecise; and, consequently, $(i i)$ the statement " $a$ is $C$ ", i.e. $a: C$, will have a truth-value in $[0,1]$ given by the degree of membership of being the individual $a$ a member of the fuzzy set $C$.

In the following, we present first some preliminaries on fuzzy set theory (for a more complete and comprehensive presentation see e.g. [7]) and then define fuzzy $\mathcal{S H O I N}(\mathrm{D})$.

\subsection{Preliminaries on Fuzzy Set Theory}

Let $X$ be a countable crisp set and let $A$ be a fuzzy subset of $X$, with membership function $\mu_{A}(x)$, or simply $A(x) \in[0,1], x \in X$. The support of $A, \operatorname{supp}(A)$, is the crisp set $\operatorname{supp}(A)=\{x \in X: A(x) \neq 0\}$. The scalar cardinality of $A,|A|$, is defined as $|A|=\sum_{x \in X} A(x)$. The fuzzy powerset of $X, \mathcal{F}(X)$, is the set of all the fuzzy sets over $X$. Let $A, B \in \mathcal{F}(X)$. We say that $A$ and $B$ are equal iff $A(x)=B(x), \forall x \in X$. $A$ is a subset of $B$ iff $A(x) \leq B(x), \forall x \in X$. We will see later on a different notion of subset, in which $A$ is a subset of $B$ to some degree in $[0,1]$. We next give the basic definitions on fuzzy set operations (complement, intersection and union).

The complement of $A, \neg A$, is given by membership function $(\neg A)(x)=n(A(x))$, for any $x \in X$. The function $n:[0,1] \rightarrow[0,1]$, called negation, has to satisfy the following conditions and extends boolean negation:

- $n(0)=1$ and $n(1)=0$;

- $\forall a, b \in[0,1], a \leq b$ implies $n(b) \leq n(a)$;

- $\forall a \in[0,1], n(n(a))=a$. 
Several negation functions have been given in the literature, e.g. Lukasiewicz negation $n_{L}(a)=1-a\left(\operatorname{syntax}, \neg_{L}\right)$ and Gödel negation $n_{G}(0)=1$ and $n(a)=0$ if $a>0$ (syntax, $\neg G$ ).

The intersection of two fuzzy sets $A$ and $B$ is given $(A \wedge B)(x)=t(A(x), B(x))$, where $t$ is a triangular norm, or simply $t$-norm. A t-norm is a function $t:[0,1] \times[0,1] \rightarrow$ $[0,1]$ that has to satisfy the following conditions:

- $\forall a \in[0,1], t(a, 1)=a ;$

- $\forall a, b, c \in[0,1], b \leq c$ implies $t(a, b) \leq t(a, c)$;

- $\forall a, b \in[0,1], t(a, b)=t(b, a)$;

- $\forall a, b, c \in[0,1], t(a, t(b, c))=t(t(a, b), c)$.

Examples of t-norms are: $t_{L}(a, b)=\max (a+b-1,0)$ (Lukasiewicz t-norm, syntax $\left.\wedge_{L}\right), t_{G}(a, b)=\min (a, b)\left(\right.$ Gödel t-norm, syntax $\left.\wedge_{G}\right)$, and $t_{P}(a, b)=a \cdot b$ (product t-norm, syntax $\left.\wedge_{P}\right)$. Note that $\forall a \in[0,1], t(a, 0)=0$.

The union of two fuzzy sets $A$ and $B$ is given $(A \vee B)(x)=s(A(x), B(x))$, where $s$ is a triangular co-norm, or simply s-norm. A s-norm is a function $s:[0,1] \times[0,1] \rightarrow$ $[0,1]$ that has to satisfy the following conditions:

$-\forall a \in[0,1], s(a, 0)=a ;$

- $\forall a, b, c \in[0,1], b \leq c$ implies $s(a, b) \leq s(a, c)$;

- $\forall a, b \in[0,1], s(a, b)=s(b, a)$;

- $\forall a, b, c \in[0,1], s(a, s(b, c))=s(s(a, b), c)$.

Examples of s-norms are: $s_{L}(a, b)=\min (a+b, 1)$ (Lukasiewicz s-norm, syntax $\left.\vee_{L}\right)$, $s_{G}(a, b)=\max (a, b)$ (Gödel s-norm, syntax $\vee_{G}$ ), and $s_{P}(a, b)=a+b-a \cdot b$ (product s-norm, syntax $\vee_{P}$ ). Note that if we consider Lukasiewicz negation, then Lukasiewicz, Gödel and product s-norm are related to their respective t-norm according to the De Morgan law: $\forall a, b \in[0,1], s(a, b)=n(t(n(a), n(b)))$.

Another important operator is implication, denoted $\rightarrow$, that gives a truth-value to the formula $A \rightarrow B$, when the truth of $A$ and $B$ are known. A fuzzy implication is a function $i:[0,1] \times[0,1] \rightarrow[0,1]$ that has to satisfy the following conditions:

- $\forall a, b, c \in[0,1], a \leq b$ implies $i(a, c) \geq i(b, c)$;

- $\forall a, b, c \in[0,1], b \leq c$ implies $i(a, b) \leq i(a, c)$;

$-\forall a \in[0,1], i(0, b)=1$;

$-\forall a \in[0,1], i(a, 1)=1$;

$-i(1,0)=0$.

In classical logic, $a \rightarrow b$ is a shorthand for $\neg a \vee b$. A generalization to fuzzy logic is, thus, $\forall a, b \in[0,1], i(a, b)=s(n(a), b)$. For instance, $\forall a, b \in[0,1], i_{K D}(a, b)=$, $\max (1-a, b)$ is the so-called Kleene-Dienes implication (syntax, $\rightarrow_{K D}$ ). Another approach to fuzzy implication is based on the so-called residuum. His formulation starts from the fact that in classical logic $\neg a \vee b$ can be re-written as $\max \{c \in\{0,1\}: a \wedge c \leq$ $b\}$. Therefore, another generalization of implication to fuzzy logic is

$$
\forall a, b \in[0,1], i(a, b)=\sup \{c \in[0,1]: t(a, c) \leq b\}
$$


For residuum based implication, $i(a, b)=1$ if $a \leq b$. If $a>b$ then, according to the chosen t-norm, we have that e.g. $i_{L}(a, b)=1-a+b$ for Lukasiewicz implication $\left(\operatorname{syntax}, \rightarrow_{L}\right), i_{G}(a, b)=b$ for Gödel implication $\left(\operatorname{syntax}, \rightarrow_{G}\right)$ ) and $i_{P}(a, b)=a / b$ for product implication (syntax, $\rightarrow_{P}$ ). Note that, for Lukasiewcz implication, s-norm and negation, we have $i_{L}(a, b)=s_{L}\left(n_{L}(a), b\right)$. The same holds using Kleene-Dienes implication, Lukasiewicz negation and Gödel s-norm. On the other hand $i_{P}(a, b) \neq$ $s_{P}\left(n_{G}(a), b\right)$ (for instance, for $0<a \leq b<1, i_{P}(a, b)=1$, while $s_{P}\left(n_{G}(a), b\right)=$ $b<1)$.

Another interesting question is when $\forall a, b \in[0,1], i(a, b)=n(t(a, n(b))$ holds, which in formulae is formulated as $a \rightarrow b \equiv \neg(a \wedge \neg b)$. It turns out that e.g., in Zadeh's logic [31] (i.e. using $\rightarrow_{K D}, \wedge_{G}, \neg_{L}$ ) this relation holds. It holds as well in the so-called Lukasiewcz logic (i.e. using $\rightarrow_{L}, \wedge_{L}, \neg_{L}$ ), while it does neither hold for Gödel logic (i.e. using $\rightarrow_{G}, \wedge_{G}, \neg_{G}$ ) nor for the product logic (i.e. using $\rightarrow_{P}, \wedge_{P}, \neg_{G}$ ). For them, just consider the case $1>a>b>0$ to verify the inequality. We will see later on that whenever $i(a, b) \neq n(t(a, n(b))$ then under the fuzzy semantics, $\forall R$. $C$ is not equivalent to $\neg \exists R . \neg C$.

Fuzzy implication can also be used to determine the degree of subset relationship between two fuzzy subsets $A$ and $B$ over $X$. Indeed, we define the degree of subsumption between $A$ and $B$, denoted $A \rightarrow B$, as $\inf _{x \in X} i(A(x), B(x))$, where $i$ is an implication function. Note that if $\forall x \in[0,1], A(x) \leq B(x)$ holds then $A \rightarrow B$ evaluates to 1 . Of course, it may be that $A \rightarrow B$ evaluates to a value $0<v<1$ as well.

We conclude the discussion on fuzzy implication by noting that we have the following inferences: assume $a \geq n$ and $i(a, b) \geq m$. Then

- under Kleene-Dienes implication we infer that if $n>1-m$ then $b \geq m$. Indeed, from $i(a, b)=\max (1-a, b) \geq m$, either $1-a \geq m$ or $b \geq m$. But $a \geq n$, so $1-a \geq m$ implies $1-m \geq a \geq n>1-m$, a contradiction. Therefore, $b \geq m$ must hold.

- under residuum based implication w.r.t. a t-norm $t$, we infer that $b \geq t(n, m)$. Indeed, from $i(a, b)=\sup \{c: t(a, c) \leq b\} \geq m$ and $a \geq n$ we have $t(n, m) \leq$ $t(n, c) \leq t(a, c) \leq b$.

A (binary) fuzzy relation $R$ over two countable crisp sets $X$ and $Y$ is a function $R: X \times$ $Y \rightarrow[0,1]$. The inverse of $R$ is the function $R^{-1}: Y \times X \rightarrow[0,1]$ with membership function $R^{-1}(y, x)=R(x, y)$, for every $x \in X$ and $y \in Y$. The composition of two fuzzy relations $R_{1}: X \times Y \rightarrow[0,1]$ and $R_{2}: Y \times Z \rightarrow[0,1]$ is defined as $\left(R_{1} \circ\right.$ $\left.R_{2}\right)(x, z)=\sup _{y \in Y} t\left(R_{1}(x, y), R_{2}(y, z)\right)$, where $t$ is a t-norm. A fuzzy relation $R$ is said to be transitive iff $R(x, z)=(R \circ R)(x, z)$.

We conclude this part with fuzzy modifiers. Fuzzy modifiers applies to fuzzy sets to change their membership function. Well known examples are modifiers like very, more_or_less, slightly, etc. These allow us to define fuzzy sets like very(High) and slightly(Mature). Formally, a modifier, $m$, is a function $m:[0,1] \rightarrow[0,1]$. For instance, we may define very $(x)=x^{2}$, while define $\operatorname{slightly}(x)=\sqrt{x}$.

In the following, we use $\wedge, \vee, \neg$ and $\rightarrow$ in infix notation, in place of a t-norm $t$, s-norm $s$, negation $n$ and implication operator $i$. 


\subsection{Fuzzy $\mathcal{S H O I N ( D )}$}

In this section we give syntax and semantics of fuzzy $\mathcal{S H O I N}(\mathrm{D})$, using the fuzzy operators defined in the previous section. We generalize the semantics given in $[13,26,29]$.

Syntax. We have seen that $\mathcal{S H O I N}(\mathrm{D})$ allows to reason with concrete data types, such as strings and integers using so-called concrete domains. In our fuzzy approach, concrete domains may be based on fuzzy sets as well. A concrete fuzzy domain is a pair $\left\langle\Delta_{\mathrm{D}}, \Phi_{\mathrm{D}}\right\rangle$, where $\Delta_{\mathrm{D}}$ is an interpretation domain and $\Phi_{\mathrm{D}}$ is the set of concrete fuzzy domain predicates $d$ with a predefined arity $n$ and an interpretation $d^{\mathrm{D}}: \Delta_{\mathrm{D}}^{n} \rightarrow[0,1]$,

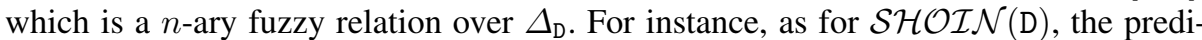
cate $\leq_{18}$ may be an unary crisp predicate over the natural numbers denoting the set of integers smaller or equal to 18 , i.e. $\leq_{18}$ : Natural $\rightarrow[0,1]$ and $\leq_{18}(x)=1$ if $x \leq 18$, $\leq_{18}(x)=0$ otherwise. So,

$$
\text { Minor }=\text { Person } \sqcap \text { ヨage. } \leq_{18}
$$

defines a person, whose age is less or equal 18, i.e. it defines a minor. On the other hand, Young: Natural $\rightarrow[0,1]$ may be a fuzzy concrete predicate over the natural numbers denoting the degree of youngness of a person's age. The concrete fuzzy predicate Young may be defined as $\operatorname{Young}(x)=\max \left(0,1-0.00075 x^{2}\right)$. So,

$$
\text { YoungPerson }=\text { Person } \sqcap \text { ヨage.Young }
$$

will denote a young person. Furthermore, by referring to Example 1, we may define the concept of sports car as the concept

$$
\text { SportsCar }=\operatorname{Car} \sqcap \exists \text { speed.very(High), }
$$

where very is a concept modifier and High is a fuzzy concrete predicate over the domain of speed expressed in kilometers per hour and may be defined as $\operatorname{High}(x)=$ $\min (1,0.004 x)$.

Similarly, we may represent "Calla is a very large, long white flower on thick stalks" as

$$
\begin{aligned}
\text { Calla }= & \text { Flower } \sqcap(\exists \text { hasSize.very }(\text { Large })) \sqcap(\exists \text { hasPetalWidth.Long }) \sqcap \\
& \sqcap(\exists \text { hasColour.White }) \sqcap(\exists \text { hasStalks.Thick }),
\end{aligned}
$$

where Large, Long and Thick are fuzzy concrete predicates.

The interesting point is that according to our semantics, e.g. a minor is likely a young person. Indeed, a minor will be a young person with degree at least $(1-0.00075$. $\left.18^{2}\right) \approx 0.76$. Informally, this value corresponds of the computation of the degree of subsumption between the two defined concepts, i.e. the degree of $\forall x$.Minor $(x) \rightarrow$ YoungPerson $(x)$, which is determined by inf $\operatorname{inatural} i\left(\leq_{18}(x)\right.$, Young $\left.(x)\right)$, where $i$ is an implication function. The fact that, as expected, a minor is a young person (to some degree) is obtained without explicitly mentioning it. This inference cannot be achieved in classical $\mathcal{S H O} \mathcal{H} \mathcal{N}(\mathrm{D})$. 
Similarly, by referring to Example 1, we will have that the car tt will be a sports car to a certain degree given by $(0.004 \cdot 243)^{2} \approx 0.94$. Therefore, unlike Example 1 , tt is now likely a sport car, as it should be.

Concerning concepts and roles, the syntax is as for $\mathcal{S H O I N}(\mathrm{D})$, except that we allow modifiers in concept expressions. That is, if $\mathrm{M}$ is a new alphabet for modifier symbols, $m \in \mathrm{M}$ is a modifier and $C$ is a $\mathcal{S H O} \mathcal{I N}(\mathrm{D})$ concept, then $m(C)$ is fuzzy $\mathcal{S H O I N}(\mathrm{D})$ concept as well. For instance, the definition of SportsCar above involves a modifier. Modifiers are allowed in fuzzy description logics such as [13, 29].

Concerning the axioms, similarly to [26], we introduce fuzzy axioms. For $n \in(0,1]$,

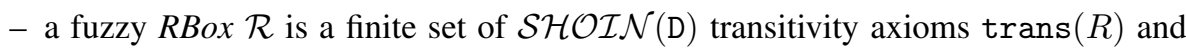
fuzzy role inclusion axioms of the form $\langle\alpha \geq n\rangle,\langle\alpha \leq n\rangle,\langle\alpha>n\rangle$ and $\langle\alpha>n\rangle$, where $\alpha$ is a $\mathcal{S H O I N}(\mathrm{D})$ role inclusion axiom;

- a fuzzy TBox $\mathcal{T}$ consists of a finite set of fuzzy concept inclusion axioms of the form $\langle\alpha \geq n\rangle,\langle\alpha \leq n\rangle,\langle\alpha>n\rangle$ and $\langle\alpha<n\rangle$ where $\alpha$ is a $\mathcal{S H O I N}(\mathrm{D})$ concept inclusion axiom $(C \sqsubseteq D)$;

- a fuzzy ABox $\mathcal{A}$ consists of a finite set of fuzzy concept and fuzzy role assertion axioms of the form $\langle\alpha \geq n\rangle,\langle\alpha \leq n\rangle,\langle\alpha>n\rangle$, or $\langle\alpha<n\rangle$, where $\alpha$ is a $\mathcal{S H O I N}$ (D) concept or role assertion. As for the crisp case, $\mathcal{A}$ may also contain a finite set of individual (in)equality axioms $a \approx b$ and $a \not b$, respectively.

For instance, $\langle a: C \geq 0.1\rangle,\langle(a, b): R \leq 0.3\rangle,\langle R \sqsubseteq S \geq 0.4\rangle$, or $\langle C \sqsubseteq D \leq 0.6\rangle$ are fuzzy axioms. Informally, from a semantics point of view, a fuzzy axiom $\langle\alpha \leq n\rangle$ constrains the membership degree of $\alpha$ to be less or equal to $n$ (similarly for $\geq,>,<$ ). For instance, $\langle j$ im:YoungPerson $\geq 0.2\rangle$, i.e. $\langle j$ im:Person $\sqcap \exists$ age.Young $\geq 0.2\rangle$, dictates that $\mathrm{jim}$ is a YoungPerson with degree at least 0.2 . On the other hand, a fuzzy concept inclusion axiom of the form $\langle C \sqsubseteq D \geq n\rangle$ dictates that the subsumption degree between $C$ and $D$ is at least $n$. A $\mathcal{S H O I N}(\mathrm{D})$ fuzzy knowledge base $\mathcal{K}=\langle\mathcal{T}, \mathcal{R}, \mathcal{A}\rangle$ consists of a fuzzy TBox $\mathcal{T}$, a fuzzy $\operatorname{RBox} \mathcal{R}$, and a fuzzy ABox $\mathcal{A}$.

Semantics. The semantics extends [26]. The main idea is that concepts and roles are interpreted as fuzzy subsets of an interpretation's domain. Therefore, $\mathcal{S H O I N}(\mathrm{D})$ axioms, rather being satisfied (true) or unsatisfied (false) in an interpretation, become a degree of truth in $[0,1]$.

A fuzzy interpretation $\mathcal{I}$ with respect to a concrete domain $\mathrm{D}$ is a pair $\mathcal{I}=\left(\Delta^{\mathcal{I}},{ }^{\mathcal{I}}\right)$ consisting of a non empty set $\Delta^{\mathcal{I}}$ (called the domain), disjoint from $\Delta_{\mathrm{D}}$, and of a fuzzy interpretation function. ${ }^{\mathcal{I}}$ that assigns

- to each abstract concept $C \in \mathrm{C}$ a function $C^{\mathcal{I}}: \Delta^{\mathcal{I}} \rightarrow[0,1]$;

- to each abstract role $R \in \mathrm{R}_{a}$ a function $R^{\mathcal{I}}: \Delta^{\mathcal{I}} \times \Delta^{\mathcal{I}} \rightarrow[0,1]$;

- to each abstract individual $a \in \mathrm{I}_{a}$ an element in $\Delta^{\mathcal{I}}$;

- to each concrete individual $c \in \mathrm{I}_{c}$ an element in $\Delta_{\mathrm{D}}$;

- to each concrete role $T \in \mathrm{R}_{c}$ a function $R^{\mathcal{I}}: \Delta^{\mathcal{I}} \times \Delta_{\mathrm{D}} \rightarrow[0,1]$;

- to each modifier $m \in \mathrm{M}$ a fixed function $\mathrm{m}:[0,1] \rightarrow[0,1]$;

- to each $n$-ary concrete predicate $d$ the fuzzy relation $d^{\mathrm{D}}: \Delta_{\mathrm{D}}^{n} \rightarrow[0,1]$. 
The mapping $\cdot{ }^{\mathcal{I}}$ is extended to concepts and roles as specified in the following table (where $x, y \in \Delta^{\mathcal{I}}, v \in \Delta_{\mathrm{D}}$ ):

$$
\begin{aligned}
& \top^{\mathcal{I}}(x)=1 \\
& \perp^{\mathcal{I}}(x)=0 \\
& \left(C_{1} \sqcap C_{2}\right)^{\mathcal{I}}(x)=C_{1}{ }^{\mathcal{I}}(x) \wedge C_{2}{ }^{\mathcal{I}}(x) \\
& \left(C_{1} \sqcup C_{2}\right)^{\mathcal{I}}(x)=C_{1}{ }^{\mathcal{I}}(x) \vee C_{2}{ }^{\mathcal{I}}(x) \\
& \left.(\neg C)^{\mathcal{I}}(x)=\neg C^{\mathcal{I}}(x)\right) \\
& (m(C))^{\mathcal{I}}(x)=\mathrm{m}\left(C^{\mathcal{I}}(x)\right) \\
& (\forall R . C)^{\mathcal{I}}(x)=\inf _{y \in \Delta^{\mathcal{I}}} R^{\mathcal{I}}(x, y) \rightarrow C^{\mathcal{I}}(y) \\
& (\exists R . C)^{\mathcal{I}}(x)=\sup _{y \in \Delta^{\mathcal{I}}} R^{\mathcal{I}}(x, y) \wedge C^{\mathcal{I}}(y) \\
& (\geq n S)^{\mathcal{I}}(x)=\sup _{y_{1}, \ldots y_{n} \in \Delta^{\mathcal{I}}} \bigwedge_{i=1}^{n} S^{\mathcal{I}}\left(x, y_{i}\right) \\
& (\leq n S)^{\mathcal{I}}(x)=\neg(\geq n+1 S)^{\mathcal{I}}(x) \\
& \left\{a_{1}, \ldots, a_{n}\right\}^{\mathcal{I}}(x)=\bigvee_{i=1}^{n} a_{i}{ }^{\mathcal{I}}=x \\
& d(v)=d^{\mathrm{D}}(v) \\
& \left\{c_{1}, \ldots, c_{n}\right\}^{\mathcal{I}}(v)=\bigvee_{i=1}^{n} c_{i}^{\mathcal{I}}=v \\
& \left(\forall T_{1}, \ldots, T_{n} . D\right)^{\mathcal{I}}(x)=\inf _{y_{1}, \ldots, y_{n} \in \Delta_{\mathrm{D}} \mathcal{I}}\left(\bigwedge_{i=1}^{n} T_{i}^{\mathcal{I}}\left(x, y_{i}\right)\right) \rightarrow D^{\mathcal{I}}\left(y_{1}, \ldots, y_{n}\right) \\
& \left(\exists T_{1}, \ldots, T_{n} . D\right)^{\mathcal{I}}(x)=\sup _{y_{1}, \ldots, y_{n} \in \Delta_{\mathrm{D}} \mathcal{I}}\left(\bigwedge_{i=1}^{n} T_{i}^{\mathcal{I}}\left(x, y_{i}\right)\right) \wedge D^{\mathcal{I}}\left(y_{1}, \ldots, y_{n}\right) \\
& \left(S^{-}\right)^{\mathcal{I}}(x, y)=S^{\mathcal{I}}(y, x) \text {. }
\end{aligned}
$$

We comment briefly some points. The semantics of $\exists R . C$

$$
(\exists R . C)^{\mathcal{I}}(d)=\sup _{y \in \Delta^{\mathcal{I}}} R^{\mathcal{I}}(x, y) \wedge C^{\mathcal{I}}(y)
$$

is the result of viewing $\exists R . C$ as the open first order formula $\exists y \cdot F_{R}(x, y) \wedge F_{C}(y)$ (where $F$ is the obvious translation of roles and concepts into First-Order Logic-FOL) and the existential quantifier $\exists$ is viewed as a disjunction over the elements of the domain. Similarly,

$$
(\forall R . C)^{\mathcal{I}}(x)=\inf _{y \in \Delta^{\mathcal{I}}} R^{\mathcal{I}}(x, y) \rightarrow C^{\mathcal{I}}(y)
$$

is related to the open first order formula $\forall y \cdot F_{R}(x, y) \rightarrow F_{C}(y)$, where the universal quantifier $\forall$ is viewed as a conjunction over the elements of the domain. However, as we already pointed out in Section 3.1, unlike the classical case, in general we do not have that $(\forall R . C)^{\mathcal{I}}=(\neg \exists R . \neg C)^{\mathcal{I}}$. If the t-norm and negation are chosen such that $\forall a, b \in[0,1], i(a, b)=n(t(a, n(b))$ holds, i.e. in formulae $a \rightarrow b \equiv \neg(a \wedge \neg b)$, then $(\forall R . C)^{\mathcal{I}}=(\neg \exists R . \neg C)^{\mathcal{I}}$ holds.

Another point concerns the semantics of number restrictions. The semantics of the concept $(\geq n S)$

$$
(\geq n S)^{\mathcal{I}}(x)=\sup _{y_{1}, \ldots y_{n} \in \Delta^{\mathcal{I}}} \bigwedge_{i=1}^{n} S^{\mathcal{I}}\left(x, y_{i}\right)
$$

is the result of viewing $(\geq n S)$ as the open first order formula

$$
\exists y_{1}, \ldots, y_{n} \cdot \bigwedge_{i=1}^{n} F_{S}\left(x, y_{i}\right) \wedge \bigwedge_{1 \leq i<j \leq n} y_{i} \neq y_{j}
$$


That is, there are at least $n$ distinct elements that satisfy to some degree $F_{R}\left(x, y_{i}\right)$. This guarantees us that $\exists S . \top \equiv(\geq 1 S)$. The semantics of $(\leq n S)$ is defined in such a way to guarantee the classical relationship $(\leq n S) \equiv \neg(\geq n+1 S)$.

An alternative definition for the $(\geq n S)$ and the $(\leq n S)$ constructs may rely on the scalar cardinality of a fuzzy set. However, we prefer to stick on the formulation, which derives directly from its FOL translation.

Finally, the mapping ${ }^{\mathcal{I}}$ is extended to non-fuzzy axioms as specified in the following table (where $a, b \in \mathrm{I}_{a}$ ):

$$
\begin{aligned}
(R \sqsubseteq S)^{\mathcal{I}} & =\inf _{x, y \in \Delta^{\mathcal{I}}} R^{\mathcal{I}}(x, y) \rightarrow S^{\mathcal{I}}(x, y) \\
(T \sqsubseteq U)^{\mathcal{I}} & =\inf _{x, y \in \Delta^{\mathcal{I}}} T^{\mathcal{I}}(x, y) \rightarrow U^{\mathcal{I}}(x, y) \\
(C \sqsubseteq D)^{\mathcal{I}} & =\inf _{x \in \Delta^{\mathcal{I}}} C^{\mathcal{I}}(x) \rightarrow D^{\mathcal{I}}(x) \\
(a: C)^{\mathcal{I}} & =C^{\mathcal{I}}\left(a^{\mathcal{I}}\right) \\
((a, b): R)^{\mathcal{I}} & =R^{\mathcal{I}}\left(a^{\mathcal{I}}, b^{\mathcal{I}}\right) .
\end{aligned}
$$

Note here that e.g. the semantics of a concept inclusion axiom $C \sqsubseteq D$ is derived directly from its FOL translation, which is of the form $\forall x \cdot F_{C}(x) \rightarrow F_{D}(x)$. This definition is novel and is clearly different from the approaches in which $C \sqsubseteq D$ is viewed as $\forall x . C(x) \leq D(x)$. This latter approach has the effect that the subsumption relationship is a classical $\{0,1\}$ relationship, while the former has the advantage that subsumption is determined up to a certain degree in $[0,1]$.

The notion of satisfiability of a fuzzy axiom $E$ by a fuzzy interpretation $\mathcal{I}$, denoted $I \models E$, is defined as follows: $\mathcal{I} \models \operatorname{trans}(R)$, iff $\forall x, y \in \Delta^{\mathcal{I}} \cdot R^{\mathcal{I}}(x, y) \geq$ $\sup _{z \in \Delta^{\mathcal{I}}} R^{\mathcal{I}}(x, z) \wedge R^{\mathcal{I}}(z, y) . I \models\langle\alpha \geq n\rangle$, where $\alpha$ is a role inclusion or concept inclusion axiom, iff $\alpha^{\mathcal{I}} \geq n$. Similarly, for the other relations $\leq,\langle$ and $>$. $I=\langle\alpha \geq n\rangle$, where $\alpha$ is a concept or a role assertion axiom, iff $\alpha^{\mathcal{I}} \geq n$. Similarly, for the other relations $\leq,<,>$. Finally, $\mathcal{I}=a \approx b$ iff $a^{\mathcal{I}}=b^{\mathcal{I}}$ and $\mathcal{I}=a \not b$ iff $a^{\mathcal{I}} \neq b^{\mathcal{I}}$.

For a set of fuzzy axioms $\mathcal{E}$, we say that $I$ satisfies $\mathcal{E}$ iff $I$ satisfies each element in $\mathcal{E}$. If $I \models E$ (resp. $I \models \mathcal{E}$ ) we say that $I$ is a model of $E$ (resp. $\mathcal{E}$ ). I satisfies (is a model of) a fuzzy knowledge base $\mathcal{K}=\langle\mathcal{T}, \mathcal{R}, \mathcal{A}\rangle$, denoted $I \models \mathcal{K}$, iff $I$ is a model of each component $\mathcal{T}, \mathcal{R}$ and $\mathcal{A}$, respectively. A fuzzy axiom $E$ is a logical consequence of a knowledge base $\mathcal{K}$, denoted $\mathcal{K} \models E$ iff every model of $\mathcal{K}$ satisfies $E$.

Example 2. Let us consider Example 1, where all axioms of the TBox and ABox are asserted with degree 1 , i.e. are of the form $\langle\alpha \geq 1\rangle$. We replace the definition of SportsCar with Definition (3) and replace the assertion involving mgb with

$$
\left\langle\text { mgb:Roadster } \sqcap(\exists \text { maker. }\{\text { mg }\}) \sqcap\left(\exists \text { speed. } \leq_{170 \mathrm{~km} / \mathrm{h}}\right) \geq 1\right\rangle .
$$

Then we have that

$$
\begin{array}{ll}
\mathcal{K} \models\langle\text { SportsCar } \sqsubseteq \operatorname{Car} \geq 1\rangle & \mathcal{K} \models\langle\text { mgb:SportsCar } \leq 0.46\rangle \\
\mathcal{K} \models\langle\text { enzo:SportsCar } \geq 1\rangle & \mathcal{K} \models\langle\text { tt:SportsCar } \geq 0.94\rangle .
\end{array}
$$

Note how the maximal speed limit of the mgb car $\left(\leq_{170 \mathrm{~km} / \mathrm{h}}\right)$ induces an upper limit, 0.46 , of the membership degree. Neither this inference is possible in classical $\mathcal{S H O I N}(\mathrm{D})$, nor the one involving tt. 
Example 3. Consider the knowledge base $\mathcal{K}$ with Definitions (1) and (2). Then we have that

$$
\mathcal{K} \models\langle\text { Minor } \sqsubseteq \text { YoungPerson } \geq 0.76\rangle,
$$

which is a relationship not captured with classical $\mathcal{S H O I N}(\mathrm{D})$.

Finally, given $\mathcal{K}$ and an axiom $\alpha$, where $\alpha$ is neither a transitivity axiom, nor an individual (in) equality axiom, it is of interest to compute $\alpha$ 's best lower and upper degree value bounds. The greatest lower bound of $\alpha$ w.r.t. $\mathcal{K}($ denoted $g l b(\mathcal{K}, \alpha))$ is

$$
g l b(\mathcal{K}, \alpha)=\sup \{n: \mathcal{K} \models\langle\alpha \geq n\rangle\},
$$

while the least upper bound of $\alpha$ with respect to $\mathcal{K}($ denoted $l u b(\mathcal{K}, \alpha)$ is

$$
\operatorname{lub}(\mathcal{K}, \alpha)=\inf \{n: \mathcal{K} \models\langle\alpha \leq n\rangle\}
$$

where $\sup \emptyset=0$ and $\inf \emptyset=1$. Determining the $l u b$ and the $g l b$ is called the Best Degree Bound (BDB) problem. For instance, the entailments in Examples 2 and 3 are the best possible degree bounds. Furthermore, note that,

$$
\operatorname{lub}(\Sigma, a: C)=\neg g l b(\Sigma, a: \neg C),
$$

i.e. the $l u b$ can be determined through the $g l b$ (and vice-versa). Similarly, $\operatorname{lub}(\Sigma,(a, b): R)=\neg g l b(\Sigma, a: \neg \exists R .\{b\})$ holds. Also, note that, $\Sigma \models\langle\alpha \geq n\rangle$ iff $\operatorname{glb}(\Sigma, \alpha) \geq n$, and similarly $\Sigma \models\langle\alpha \leq n\rangle$ iff $\operatorname{lub}(\Sigma, \alpha) \leq n$ hold.

Concerning the entailment problem, it is quite easily verified that, as for the crisp case, the entailment problem can be reduced to the unsatisfiability problem:

$$
\begin{aligned}
& \langle\mathcal{T}, \mathcal{R}, \mathcal{A}\rangle \models\langle\alpha \geq n\rangle \text { iff }\langle\mathcal{T}, \mathcal{R}, \mathcal{A} \cup\{\langle\alpha<n\rangle\}\rangle \text { is not satisfiable } \\
& \langle\mathcal{T}, \mathcal{R}, \mathcal{A}\rangle \models\langle\alpha \leq n\rangle \text { iff }\langle\mathcal{T}, \mathcal{R}, \mathcal{A} \cup\{\langle\alpha>n\rangle\}\rangle \text { is not satisfiable } .
\end{aligned}
$$

Unfortunately, from a computational point of view, no calculus exists yet checking satisfiability of fuzzy $\mathcal{S H O I N}(\mathrm{D})$ knowledge bases. [13, 29] report a calculus for the case of $\mathcal{A L C}$ [24] (with concept constructors $\top, \perp, \neg, \sqcap, \sqcup, \forall, \exists$ ) with modifiers and simple TBox, with min, max and $\rightarrow_{K D}$ connectives. No indication for the BDB problem is given. $[25,26]$ reports a calculus for $\mathcal{A L C}$ and simple TBox, with min, max and $\rightarrow K D$ connectives and addresses the BDB problem and, [27] shows how the satisfiability problem and the BDB problem can be reduced to classical $\mathcal{A L C}$ and, thus, can be resolved by means of a tools like FACT and RACER. However, despite these negative results, recently [28] reports a calculus for $\mathcal{A L C}(\mathrm{D})$ whenever the connectives, the modifiers and the concrete fuzzy predicates are representable as a bounded Mixed Integer Program. For instance, Lukasiewicz logic satisfies these conditions as well as the membership functions for concrete fuzzy predicates we have presented in this paper. Additionally, modifiers should be a combination of linear functions. In that case the calculus consists of a set of constraint propagation rules and an invocation to an oracle for bounded Mixed Integer Programming. But, indeed, the computational aspect is definitely a point that has to be addressed in forthcoming works. 


\section{Conclusions and Outlook}

We have presented a fuzzy extension of $\mathcal{S H O I N}(\mathrm{D})$ showing that its representation and reasoning capabilities go clearly beyond classical $\mathcal{S H O} \mathcal{H} \mathcal{N}(\mathrm{D})$. Interestingly, we allow modifiers, fuzzy concrete domain predicates and fuzzy axioms to appear in a $\mathcal{S H O I N}(\mathrm{D})$ knowledge base and the entailment and the subsumption relationship hold to a certain degree. To the best of our knowledge, no other work has yet extended the semantics to $\mathcal{S H O I N}$ (D) in such a way. The argument supporting the necessity of such an extension relies on the fact that vague concepts are abundant in human knowledge and, thus, appear likely in Web content.

The main direction for future work involves the computational aspect. Currently, we are addressing the fundamental issue to develop a calculus for reasoning within $\mathcal{A L C}(\mathrm{D})$, i.e. $\mathcal{A} \mathcal{L C}$ with concrete domains and arbitrary t-norm, co-norm, negation and residuum as implication. We are investigating the possibility to use the methods developed in the context of Many-Valued Logics [12], which seem to particularly well-suited to our context. These procedures have then to be combined with a procedure to deal with fuzzy concrete domains, for which we plan to rely on [18].

Another direction is in extending fuzzy $\mathcal{S H O I N}(\mathrm{D})$ with fuzzy quantifiers, where the $\forall$ and $\exists$ quantifiers are replaced with fuzzy quantifiers like most, some, usually and the like (see [23] for a preliminary work in this direction). This allows to define concepts like

$$
\begin{aligned}
& \text { TopCustomer }=\text { Customer } \sqcap \text { (Usually)buys.ExpensiveItem } \\
& \text { ExpensiveItem }=\text { Item } \sqcap \exists \text { price.High } .
\end{aligned}
$$

Here, the fuzzy quantifier Usually replaces the classical quantifier $\forall$ and High is a fuzzy concrete predicate.

Fuzzy quantifiers can be applied to inclusion axioms as well, allowing to express, for instance:

$$
\text { (Most)Bird } \sqsubseteq \text { FlyingObject . }
$$

Here the fuzzy quantifier Most replaces the classical universal quantifier $\forall$ assumed in the inclusion axioms. The above axiom allows to state that most birds fly.

Ultimately, we believe that the fuzzy extension of $\mathcal{S H O I N}(\mathrm{D})$ is of great interest to the Semantic Web community, as it allows to express naturally a wide range of concepts of actual domains, for which a classical $\mathcal{S H O I N}(\mathrm{D})$ representation is unsatisfactory.

\section{References}

1. Franz Baader and Philipp Hanschke. A schema for integrating concrete domains into concept languages. In Proc. of the 12th Int. Joint Conf. on Artificial Intelligence (IJCAI-91), pages 452-457, Sydney, 1991.

2. Franz Baader, Diego Calvanese, Deborah McGuinness, Daniele Nardi, and Peter F. PatelSchneider, editors. The Description Logic Handbook: Theory, Implementation, and Applications. Cambridge University Press, 2003.

3. Fahiem Bacchus. Representing and Reasoning with Probabilistic Knowledge. The MIT Press, 1990. 
4. T. Berners-Lee, J. Hendler, and O. Lassila. The semantic web. The Scientific American, 284(5):34-43, 2001.

5. P. Bonatti and A. Tettamanzi. Some complexity results on fuzzy description logics. In A. Petrosino V. Di Gesù, F. Masulli, editor, WILF 2003 Int. Workshop on Fuzzy Logic and Applications, LNCS 2955, Berlin, 2004. Springer Verlag.

6. Rita Maria da Silva, Antonio Eduardo C. Pereira, and Marcio Andrade Netto. A system of knowledge representation based on formulae of predicate calculus whose variables are annotated by expressions of a fuzzy terminological logic. In Proc. of the 5th Int. Conf. on Information Processing and Managment of Uncertainty in Knowledge-Based Systems, (IPMU-94), LNCS 945. Springer-Verlag, 1994.

7. Didier Dubois and Henri Prade. Fuzzy Sets and Systems. Academic Press, New York, NJ, 1980.

8. Didier Dubois and Henri Prade. Approximate and commonsense reasoning: From theory to practice. In Zbigniew W. Ras and Michalewicz Maciek, editors, Proc. of the 9th Int. Sym. on Methodologies for Intelligent Systems (ISMIS-96), LNAI 1079, pages 19-33. SpringerVerlag, 1996.

9. Pan et al. Specification of coordination of rule and ontology languages. Technical report, Knowledgeweb Network of Excellence, EU-IST-2004-507482, 2004. Deliverable D2.5.1.

10. N. Guarino and R. Poli. Formal ontology in conceptual analysis and knowledge representation. Int. Journal of Human and Computer Studies, 43(5/6):625-640, 1995.

11. Volker Haarslev and Ralf Möller. RACER system description. In Proc. of Int. Joint Conf. on Automated Reasoning (IJCAR-01), LNAI 2083, pages 701-705, 2001. Springer.

12. Reiner Hänle and Gonzalo Escalada-Imaz. Deduction in many-valued logics: a survey. Mathware and Soft Computing, IV(2):69-97, 1997.

13. Steffen Hölldobler, Hans-Peter Störr, and Tran Dinh Khang. A fuzzy description logic with hedges and concept modifiers. In Proc. of the 10th Int. Conf. on Information Processing and Managment of Uncertainty in Knowledge-Based Systems, (IPMU-04), 2004.

14. Ian Horrocks. Using an expressive description logic: Fact or fiction? In Proc. of the 8th Int. Conf. on the Principles of Knowledge Representation and Reasoning (KR-98), 1998.

15. Ian Horrocks, Peter F. Patel-Schneider, and Frank van Harmelen. From SHIQ and RDF to OWL: The making of a web ontology language. Journal of Web Semantics, 1(1):7-26, 2003.

16. Ian Horrocks, Peter F. Patel-Schneider. Reducing OWL entailment to description logic satisfiability. Journal of Web Semantics, 2004.

17. R. Kruse, E. Schwecke, and J. Heinsohn. Uncertainty and Vagueness in Knowledge Based Systems. Springer-Verlag, Berlin, Germany, 1991.

18. C. Lutz. Description logics with concrete domains-a survey. In Advances in Modal Logics Volume 4. King's College Publications, 2003.

19. C. Lutz, F. Wolter, and M. Zakharyaschev. A tableau algorithm for reasoning about concepts and similarity. In Proc. of the 12th Int. Conf. on Automated Reasoning with Analytic Tableaux and Related Methods TABLEAUX 2003, number 2796 in LNAI, Rome,Italy, 2003. Springer.

20. Carsten Lutz. Reasoning with concrete domains. In Proc. of the 16th Int. Joint Conf. on Artificial Intelligence, pages 90-95. Morgan Kaufmann Publishers Inc., 1999.

21. Carsten Lutz. Nexp time-complete description logics with concrete domains. ACM Trans. Comput. Logic, 5(4):669-705, 2004.

22. J. Pearl. Probabilistic Reasoning in Intelligent Systems: Networks of Plausible Inference. Morgan Kaufmann, Los Altos, 1988.

23. D Sánchez and G.B. Tettamanzi. Generalizing quantification in fuzzy description logics. In Proc. of the 8th Fuzzy Days in Dortmund, 2004.

24. Manfred Schmidt-Schauß and Gert Smolka. Attributive concept descriptions with complements. Artificial Intelligence, 48:1-26, 1991. 
25. Umberto Straccia. A fuzzy description logic. In Proc. of the 15th Nat. Conf. on Artificial Intelligence (AAAI-98), pages 594-599, Madison, USA, 1998.

26. Umberto Straccia. Reasoning within fuzzy description logics. Journal of Artificial Intelligence Research, 14:137-166, 2001.

27. Umberto Straccia. Transforming fuzzy description logics into classical description logics. In Proc. of the 9th European Conf. on Logics in Artificial Intelligence (JELIA-04), LNCS 3229, pages 385-399, 2004. Springer Verlag.

28. Umberto Straccia. Fuzzy description logics with concrete domains. Technical Report 2005-TR-03, Istituto di Scienza e Tecnologie dell'Informazione, Consiglio Nazionale delle Ricerche, Pisa, Italy, 2005.

29. C. Tresp and R. Molitor. A description logic for vague knowledge. In Proc. of the 13th European Conf. on Artificial Intelligence (ECAI-98), Brighton (England), August 1998.

30. John Yen. Generalizing term subsumption languages to fuzzy logic. In Proc. of the 12th Int. Joint Conf. on Artificial Intelligence (IJCAI-91), pages 472-477, 1991.

31. L. A. Zadeh. Fuzzy sets. Information and Control, 8(3):338-353, 1965. 